\title{
Towards a Unified Understanding of Jet Quenching and Elliptic Flow within Perturbative QCD Parton Transport
}

\author{
Oliver Fochler, ${ }^{1}$ Zhe $\mathrm{Xu},{ }^{1}$ and Carsten Greiner $^{1}$ \\ ${ }^{1}$ Institut für Theoretische Physik, Goethe-Universität Frankfurt am Main \\ Max-von-Laue-Straße 1, D-60438 Frankfurt am Main, Germany
}

\begin{abstract}
The gluonic contribution to the nuclear modification factor $R_{A A}$ is investigated for central Au $+\mathrm{Au}$ collisions at $\sqrt{s}=200 \mathrm{AGeV}$ employing a perturbative QCD-based parton cascade including radiative processes. A flat quenching pattern is found up to transverse momenta of $30 \mathrm{GeV}$, which is slightly smaller compared with results from the Gyulassy-Levai-Vitev formalism. We demonstrate that the present microscopic transport description provides a challenging means of investigating both jet quenching and a strong buildup of elliptic flow in terms of the same standard perturbative QCD interactions.
\end{abstract}

PACS numbers: 12.38.Mh,24.10.Lx,24.85.+p,25.75.-q

The phenomenon of jet quenching [1] has been one of the two striking discoveries at the Relativistic Heavy Ion Collider (RHIC) [2, 3]. It is widely believed to provide tomography of the quark-gluon plasma (QGP) created in ultrarelativistic heavy ion collisions. Furthermore, the strong elliptic flow, quantified by the Fourier parameter $v_{2}$, explores the early bulk properties of the medium. Since ideal hydrodynamics can fairly reproduce the observed $v_{2}$-dependence on centrality [4], viscosity in the QGP is believed to be small [5, [6]. Hence, the QGP behaves like a perfect fluid, which has been the second major discovery at RHIC. So far, it has not been possible to relate both phenomena by a common understanding of the underlying microscopic processes.

Early attempts to describe the collective behavior by means of standard binary, elastic perturbative QCD (pQCD) collisions within a parton cascade have failed unless (unphysically) large cross sections are employed [7, 8, 9]. With such cross sections, as we will also show in the following, the attenuation of jets would be far too strong, basically extinguishing all high-momentum partons. There exist several related theoretical schemes of the energy loss on the partonic level based on radiative pQCD interactions that are aimed at describing the observed amount of jet quenching 10, 11, 12, 13, 14, 15, 16. In particular, the energy loss is found to be dominantly radiative. It is thus believed that different microscopic physics become manifest in perturbative interactions of jets with bulk particles on the one hand, and in seemingly much stronger interactions between medium constituents that give rise to the liquid-like bulk properties on the other hand.

It has been recently demonstrated within BAMPS (A Boltzmann approach to multi parton scatterings) [17, 18], a sophisticated kinetic pQCD based parton cascade including radiative contributions, that inelastic Bremsstrahlung processes can drive the system to equilibration within a short time less than $1 \mathrm{fm} / c$. Moreover, pQCD Bremsstrahlung processes generate a sizeable de- gree of collective flow [19] and a small ratio of shear viscosity to entropy density $\eta / s$. Using parton-hadron duality the simulated elliptic flow coefficient $v_{2}$ has been demonstrated to be in fair agreement with data employing a coupling constant $\alpha_{s}=0.3 \div 0.6$. Some details on freeze-out [20], hadronization and possible hadronic phase contributions have still to be addressed. For the created gluon plasma $\eta / s$ is in the range $0.08 \div 0.15$, supporting the idea that the medium behaves like a nearly ideal fluid [19, 21].

The present study is motivated by the challenging question whether the employed treatment of pQCD interactions, including radiative processes, can simultaneously account for the quenching of high-momentum partons.

Microscopic transport calculations provide a realistic way of understanding heavy ion collisions including the full dynamics of the system and allowing for non-thermal initial conditions. We employ BAMPS to investigate the evolution of gluon matter produced in heavy ion collisions at RHIC. Treating gluons as semi-classical and massless Boltzmann particles, elastic $g g \rightarrow g g$ interactions are included via the leading order $\mathrm{pQCD}$ differential cross section $\frac{d \sigma_{g g \rightarrow g g}}{d q_{\perp}^{2}}=\frac{9 \pi \alpha_{s}^{2}}{\left(\mathbf{q}_{\perp}^{2}+m_{D}^{2}\right)^{2}}$. The effective Bremsstrahlung matrix element

$$
\begin{aligned}
\left|\mathcal{M}_{g g \rightarrow g g g}\right|^{2}= & \frac{72 \pi^{2} \alpha_{s}^{2} s^{2}}{\left(\mathbf{q}_{\perp}^{2}+m_{D}^{2}\right)^{2}} \frac{48 \pi \alpha_{s} \mathbf{q}_{\perp}^{2}}{\mathbf{k}_{\perp}^{2}\left[\left(\mathbf{k}_{\perp}-\mathbf{q}_{\perp}\right)^{2}+m_{D}^{2}\right]} \times \\
& \times \Theta\left(\frac{\Lambda_{g}}{\gamma}-\tau\right)
\end{aligned}
$$

is used for inelastic gluon multiplication and annihilation processes. $\mathbf{q}_{\perp}$ and $\mathbf{k}_{\perp}$ denote the perpendicular components of the momentum transfer and of the radiated gluon momentum in the center of mass $(\mathrm{CM})$ frame of the colliding particles, respectively. Gluon interactions are screened by a dynamically computed Debye mass $m_{D}^{2}=d_{G} \pi \alpha_{s} \int \frac{d^{3} p}{(2 \pi)^{3}} \frac{1}{p} N_{c} f$, where $d_{G}=16$ is the gluon degeneracy factor for $N_{c}=3$ and $f=f(p, x, t)$ is the local distribution function. 
In the treatment of Bremsstrahlung processes the coherent Landau, Pomeranchuk and Migdal (LPM) effect [22], leads to a suppression of the emission rate for high energy particles. Such interference effects cannot be included in the microscopic transport calculations. Therefore, the theta function in (11) is introduced to ensure that only independent processes are considered, i.e. that only processes within the so called Bethe-Heitler regime are taken into consideration. Physically this implies that the formation time $\tau$ of the emitted gluon must not exceed the mean free path of the parent particle. The mean free path $\Lambda_{g}$ is given in the local comoving frame of a computational spatial cell, which is not identical with the frame where the gluon is emitted purely transversal and thus $\tau=1 / k_{\perp}$. A Lorentz factor $\gamma=\frac{\cosh y}{\sqrt{1-\beta^{2}}}(1+\beta \tanh y \cos \theta)$ has to be employed. $\beta$ denotes the boost velocity from the comoving frame to the CM frame of the colliding particles, $y$ is the rapidity of the emitted gluon measured from the CM frame and $\theta$ is the angle between $\vec{\beta}$ and the axis of the colliding particles in the $\mathrm{CM}$ frame. For thermal energies the boost $\beta$ becomes small and the theta function reduces to $\Theta\left(k_{\perp} \Lambda_{g}-\cosh y\right)$.

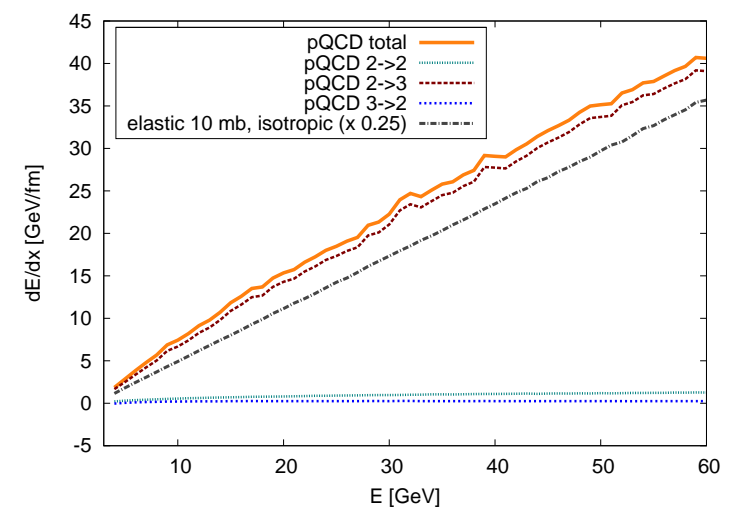

FIG. 1: (Color online) Differential energy loss of a gluon jet in a static and thermal medium of gluons with $T=400 \mathrm{MeV}$. The contributions of the different $\mathrm{pQCD}$ processes to the total $d E / d x$ are shown. Additionally $d E / d x$ scaled down by a factor of 4 is shown for a gluon jet interacting only via isotropic binary scatterings with a fixed cross section $\sigma=10 \mathrm{mb}$.

The cutoff in (1) affects the overall value of the interaction probability for $g g \leftrightarrow g g g$ as well as the momentum shape of the spectrum of the radiated gluons. Fig. 1 shows the differential energy loss $d E / d x$ of a high energy gluon jet in a static and thermal medium of gluons with temperature $T=400 \mathrm{MeV}$ as a function of the jet energy $E$. Throughout this work we employ a fixed coupling constant $\alpha_{s}=0.3$. The elastic energy loss from $g g \rightarrow g g$ interactions is found to be only slowly rising with energy to a level of $d E / d x \approx 1 \mathrm{GeV} / \mathrm{fm}$ at $E=40 \mathrm{GeV}$ and as expected the contribution from $g g g \rightarrow g g$ processes is negligible with $d E / d x \approx 0.3 \mathrm{GeV} / \mathrm{fm}$. Radiative $g g \rightarrow g g g$ processes strongly dominate the energy loss, for instance resulting in a total $d E / d x \approx 29 \mathrm{GeV} / \mathrm{fm}$ at $E=40 \mathrm{GeV}$, with $d E / d x$ almost linearly increasing with the jet energy $E$. In comparison to the Arnold-MooreYaffe formalism [14] a hard gluon with $E=40 \mathrm{GeV}$ loses $75 \%$ of its energy about a factor of 2 faster [23].

The individual cross sections moderatly increase with the jet energy, yielding a total $\left\langle\sigma_{\text {tot }}\right\rangle \approx 3.5 \mathrm{mb}$ at $E=$ $40 \mathrm{GeV}$ [24] for a medium as characterized above. We note that a dependence of $d E / d x$ on the distance the parton has propagated cannot be observed in our approach, since coherent effects are explicitely not included.

For arguments to come, Fig. 1 also shows $d E / d x$ (scaled down by a factor of 4 ) for a gluon jet interacting purely elastic with the medium at a fixed cross section of $\sigma=10 \mathrm{mb}$ and an isotropic angular distribution. The differential energy loss at this temperature is about a factor of $3 \div 4$ larger than $d E / d x$ from pQCD interactions, e.g. $d E /\left.d x\right|_{\sigma=10 \mathrm{mb}} \approx 94 \mathrm{GeV} / \mathrm{fm}$ at $E=40 \mathrm{GeV}$.

The initial gluon distribution for $\mathrm{Au}+\mathrm{Au}$ collisions at $200 \mathrm{AGeV}$ is chosen to be an ensemble of mini-jets with a lower momentum cut-off $p_{0}=1.4 \mathrm{GeV}$, produced in nucleon-nucleon collisions following a Glauber-model with a Wood-Saxon density profile and Glück-Reya-Vogt parton distribution functions [25] with a $K$-factor of 2 . Free streaming is applied to regions where the energy density has dropped below a critical energy density of $\varepsilon_{c}=1 \mathrm{GeV} / \mathrm{fm}^{3}$. With this setup it has been demonstrated [18, 19, 20] that the experimental findings for the rapidity distribution of transverse energy and the flow parameter for various centralities are nicely reproduced.

Due to the steeply falling momentum spectrum of the initial jets one would need to simulate an infeasibly high number of events in order to obtain sufficient statistics at high- $p_{T}$. We have therefore developed a suitable weighting and reconstruction scheme. The underlying idea is to simulate a huge number of initial spectra, which is rather straightforward from the computational point of view, and to select such events for further simulations that contain high- $p_{T}$ partons. The results then need to be appropriately weighted. For this we characterize each initial state according to $X=\max \left(p_{T, i}\right)$, the maximum $p_{T}$ in a given rapidity range. From each bin $j$ in $X$ a number of $N_{j}$ events is simulated, the results are averaged within these bins and finally combined with appropriate weights $P_{j}$. This procedure has been thoroughly tested [24] and, so far, allows for the investigation of observables up to $p_{T} \approx 30 \mathrm{GeV}$. In the following we use a bin-size of $1 \mathrm{GeV}$ and select up to $N_{j}=120$ events per bin for full simulation.

Jet quenching is generally specified in terms of the nuclear modification factor

$$
R_{A A}=\frac{d^{2} N_{A A} / d p_{T} d y}{T_{A A} d^{2} \sigma_{N N} / d p_{T} d y}
$$

which is the ratio of the particle yield in a $\mathrm{A}+\mathrm{A}$ collision at given $p_{T}$ and $y$ to the yield in a $\mathrm{p}+\mathrm{p}$ collision scaled by 

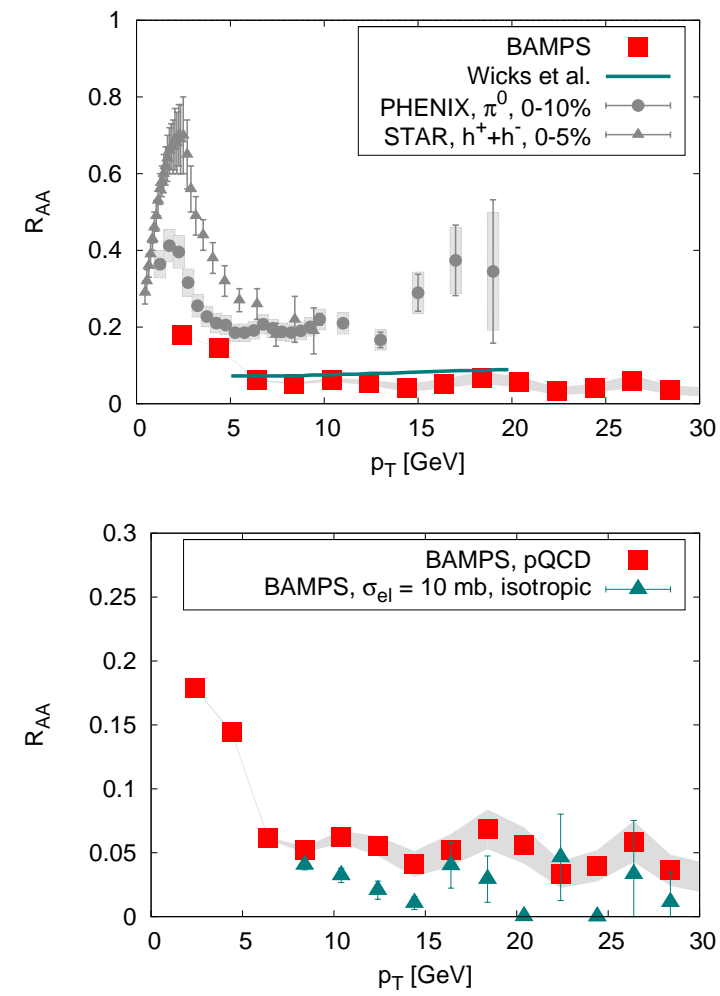

FIG. 2: (Color online) Upper: Gluonic $R_{A A}$ at midrapidity $(y \epsilon[-0.5,0.5])$ as extracted from simulations for central $\mathrm{Au}+\mathrm{Au}$ collisions at $200 \mathrm{AGeV}$. The shaded area indicates the statistical error. For direct comparison the result from Wicks et al. [16] for the gluonic contribution to $R_{A A}$ and experimental results from PHENIX [28] for $\pi^{0}$ and STAR [29] for charged hadrons are shown. Lower: Gluonic $R_{A A}$ (triangles) for a scenario where all particles with $p_{T}>8 \mathrm{GeV}$ can only interact elastically with fixed $\sigma=10 \mathrm{mb}$.

the appropriate number of binary collisions. We directly compute $R_{A A}$ by taking the ratio of the final $p_{T}$ spectra to the initial mini-jet spectra. In this Letter we concentrate on central ( $b=0 \mathrm{fm}$ ) collisions; $R_{A A}$ for non-central collisions can be straightforwardly studied and will be presented in an upcoming work [24]. Fig. 2 shows the result for the gluonic contribution to $R_{A A}$, exhibiting a clear suppression of high- $p_{T}$ gluon jets at a roughly constant level of $R_{A A}^{\text {gluons }} \approx 0.053$. This constitutes the major result of the present study.

The suppression of gluon jets is approximately a factor $3 \div 4$ stronger than seen in experimental pion data. An excessive quenching, however, was to be expected since at present the simulation does not include quarks, which are bound to lose considerably less energy due to their color factor and dominate the initially produced jets from $p_{T} \approx 20 \mathrm{GeV}$. As also discussed in [20], the inclusion of light quark degrees of freedom would yield better agreement between BAMPS results and data on particle spectra, mean- $p_{T}$ and the $p_{T}$-dependence of elliptic flow. Indeed, comparing with state of the art results from Wicks et al. [16], obtained within the GLV formalism 13], for the gluonic contribution to $R_{A A}$ (seen as the line in Fig. 2), which in their approach together with the quark contribution reproduces the experimental data, one finds better agreement. The gluonic suppression in their calculations is found to be $R_{A A} \approx 0.07 \div 0.09$. They assume a gluon density per unit rapidity of $d N_{g} / d y=1100$ and the strong expansion of the fireball has to be included via an effective description. In the present microscopic treatment the full dynamics are selfconsistently included, where the initial gluon density is given by $d N_{g} / d y \approx 700$, which then evolves to a final $d N_{g} / d y \approx 800$. The inclusion of light quarks will provide important means of further verification and will be addressed in a future work.

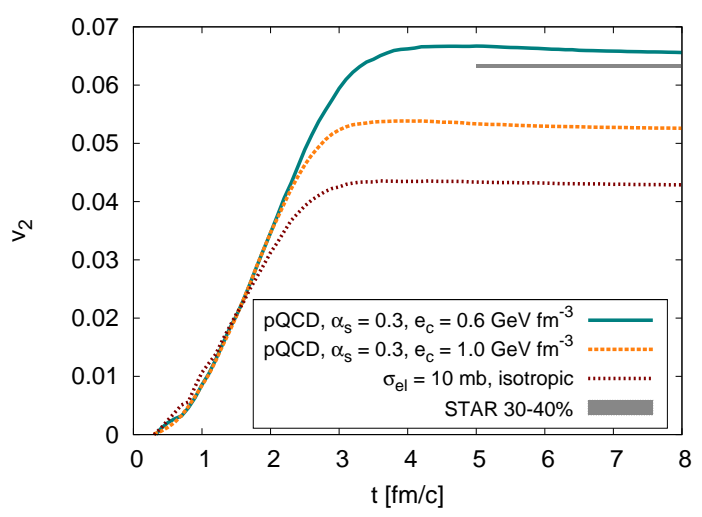

FIG. 3: (Color online) Generation of elliptic flow in BAMPS at midrapidity for a noncentral collision with an impact parameter of $b=8.6 \mathrm{fm}$, obtained from calculations with $\alpha_{s}=0.3$ and two freeze-out energy densities, $\varepsilon_{c}=$ 0.6 and $1.0 \mathrm{GeV} \mathrm{fm}^{-3}[20]$. For comparison the result employing solely binary collisions with constant $\sigma=10 \mathrm{mb}$ $\left(\varepsilon_{c}=1.0 \mathrm{GeV} \mathrm{fm}^{-3}\right)$ is shown. The grey-shaded band represents the experimental value [30].

To demonstrate the importance of inelastic processes for explaining both elliptic flow and jet quenching within a combined framework, in Fig. 3 we compare the elliptic flow for a scenario of only binary and isotropic collisions with $\sigma=10 \mathrm{mb}$ to the full results from [20]. Though the cross section is large, it still does not fully succeed in explaining the strong elliptic flow. A still larger one has to be (phenomenologically) employed in order to generate enough flow [9]. On the other hand, we also investigate a scenario in which jet-like gluons with $p_{T}>8 \mathrm{GeV}$ can only interact elastically with the same fixed and isotropic cross section of $\sigma=10 \mathrm{mb}$. In this calculation, the medium particles interact with the usual pQCD based matrix elements, including inelastic collisions. As already expected from Fig. 1, the results shown in the lower panel of Fig. 2 explicitly demonstrate that already with $\sigma=10 \mathrm{mb}$ the suppression is too strong. So, while approaches using fixed and large cross sections might be able to reproduce thermalization times, angular correlations [26] or elliptic flow [9, 27], they would fail at simultaneously reproducing the correct nuclear modifica- 
tion factor.

The use of a full microscopic transport treatment also offers the possibility to investigate observables, that are not directly accessible in experiment. Fig. 4 shows the probability distribution of production points in the transverse plane of gluon jets with a final $p_{T}>10 \mathrm{GeV}$ in a central $(b=0 \mathrm{fm}) \mathrm{Au}+\mathrm{Au}$ collision using the full pQCD BAMPS version. Events in this illustration are rotated such that the jets emerge with their final $\vec{p}_{T}$ parallel to the $x$-axis and directed in positive $x$-direction. The spatial extent of the initial production points is roughly controlled by the parameter $R_{A}$ in the Wood-Saxon distribution $n_{A}(r)=n_{0} /\left(1+e^{\left(r-R_{A}\right) / d}\right)$, with $R_{A}=6.37 \mathrm{fm}$ in our case. A bias towards production points near the surface is visible as would be naively expected for the strong jet suppression seen in Fig. 2. The escaping jets have been produced on the average $1.7 \mathrm{fm}$ away from the surface and have undergone $\left\langle N_{23}\right\rangle \approx 0.6$ radiative processes $\left(\left\langle N_{22}\right\rangle \approx 2.0\right.$ and $\left.\left\langle N_{32}\right\rangle \approx 0.1\right)$. Still, at such high transverse momenta a few jets can indeed probe the interior of the reaction volume, corroborating the notion that the inclusion of the full medium dynamics is crucial to the investigation of jet quenching.

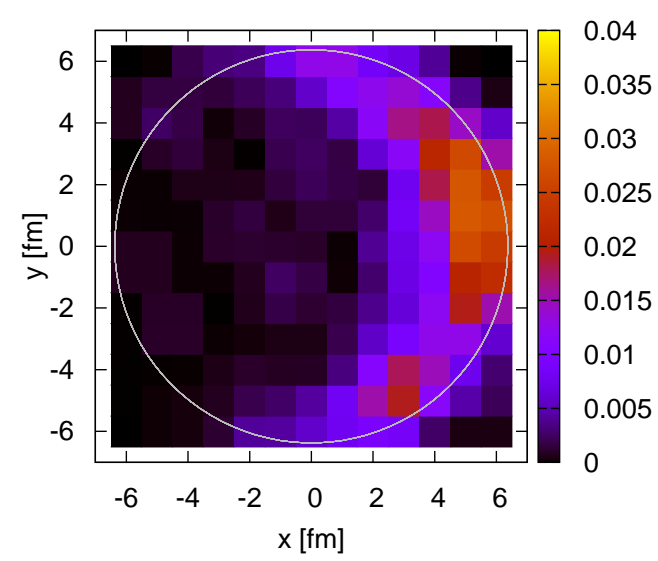

FIG. 4: (Color online) Probability distribution of production points of jets with final $p_{T}>10 \mathrm{GeV}$. Events are rotated such that jets in the final state travel in the positive $x$-direction.

For the first time a consistent and fully pQCD based microscopic parton transport description has been applied as a common setup to both elliptic flow and jet quenching observed in RHIC experiments. As established previously, the gluon matter simulated in the parton cascade BAMPS exhibits a sizeable pressure build-up and a small ratio of shear viscosity to entropy, $\eta / s$. In this work we found that the suppression of high $-p_{T}$ gluon jets is roughly constant at $R_{A A}^{\text {gluons }} \approx 0.053$ over a large $p_{T}$ range, with inelastic $g g \leftrightarrow g g g$ processes being the main cause of energy loss. The observed level of jet quenching is in reasonable agreement with recent analytic results, though the suppression of gluonic jets appears to be somewhat stronger. Future studies including light quarks and a fragmentation scheme into the simulation will help to further confirm our findings that bulk properties of the medium and the energy loss of high $-p_{T}$ partons can be described within a common transport approach. The investigation of elliptic flow at large transverse momenta will also provide important means of cross-checking.

The authors would like to thank M. Gyulassy for stimulating discussions throughout this work. The simulations were performed at the Center for Scientific Computing of the Goethe University Frankfurt.

[1] M. Gyulassy and X.-N. Wang, Nucl. Phys. B420, 583 (1994).

[2] C. Adler et al. (STAR), Phys. Rev. Lett. 89, 202301 (2002).

[3] K. Adcox et al. (PHENIX), Phys. Rev. Lett. 88, 022301 (2002).

[4] P. Huovinen, P. F. Kolb, U. W. Heinz, P. V. Ruuskanen, and S. A. Voloshin, Phys. Lett. B503, 58 (2001), hep$\mathrm{ph} / 0101136$.

[5] L. P. Csernai, J. I. Kapusta, and L. D. McLerran, Phys. Rev. Lett. 97, 152303 (2006).

[6] R. A. Lacey et al., Phys. Rev. Lett. 98, 092301 (2007).

[7] B. Zhang, M. Gyulassy, and C. M. Ko, Phys. Lett. B455, 45 (1999)

[8] Z.-w. Lin and C. M. Ko, Phys. Rev. C65, 034904 (2002).

[9] D. Molnar and M. Gyulassy, Nucl. Phys. A697, 495 (2002); D. Molnar and P. Huovinen, Phys. Rev. Lett. 94, 012302 (2005).

[10] B. G. Zakharov, JETP Lett. 63, 952 (1996).

[11] R. Baier, Y. L. Dokshitzer, A. H. Mueller, S. Peigne, and D. Schiff, Nucl. Phys. B484, 265 (1997).

[12] R. Baier, Y. L. Dokshitzer, A. H. Mueller, and D. Schiff, Phys. Rev. C58, 1706 (1998).

[13] M. Gyulassy, P. Levai, and I. Vitev, Nucl. Phys. B594, 371 (2001).

[14] S. Jeon and G. D. Moore, Phys. Rev. C71, 034901 (2005).

[15] C. A. Salgado and U. A. Wiedemann, Phys. Rev. D68, 014008 (2003).

[16] S. Wicks, W. Horowitz, M. Djordjevic, and M. Gyulassy, Nucl. Phys. A784, 426 (2007).

[17] Z. Xu and C. Greiner, Phys. Rev. C71, 064901 (2005).

[18] Z. Xu and C. Greiner, Phys. Rev. C76, 024911 (2007).

[19] Z. Xu, C. Greiner, and H. Stocker, Phys. Rev. Lett. 101, 082302 (2008).

[20] Z. Xu and C. Greiner, Phys. Rev. C79, 014904 (2009).

[21] Z. Xu and C. Greiner, Phys. Rev. Lett. 100, 172301 (2008), 0710.5719.

[22] A. B. Migdal, Phys. Rev. 103, 1811 (1956).

[23] B. Schenke, priv. comm. (2009).

[24] O. Fochler, Z. Xu, and C. Greiner, forthcoming (2009).

[25] M. Gluck, E. Reya, and A. Vogt, Z. Phys. C67, 433 (1995).

[26] G. L. Ma et al., Phys. Lett. B647, 122 (2007).

[27] B. Zhang, L.-W. Chen, and C.-M. Ko, Phys. Rev. C72, 024906 (2005).

[28] A. Adare et al. (PHENIX), Phys. Rev. Lett. 101, 232301 (2008).

[29] J. Adams et al. (STAR), Phys. Rev. Lett. 91, 172302 (2003). 
[30] J. Adams et al. (STAR), Phys. Rev. C72, 014904 (2005). 